See Article page 13.

\section{Commentary: Do we really need specific recommendations for the use of one-piece hybrid devices?}

\author{
Ourania Preventza, MD, and \\ Davut Cekmecelioglu, MD
}

This is an interesting descriptive study ${ }^{1}$ in a small sample of 36 patients who underwent a single-stage frozen elephant trunk (FET) procedure with the E-vita OPEN device or the Thoraflex device. During 26 months' follow-up, $28 \%$ of patients had endoleak and $8 \%$ had sac expansion. Patients without endoleak or expansion more often had stents that were $>10 \%$ oversize and had a $>30$-mm distal seal. The authors reported 13 endoleak events, which prevented meaningful analysis, and the series covers a long period of time. Nevertheless, this manuscript ${ }^{1}$ brings to mind 2 important topics: single-stage FET using one of the newer hybrid devices, and endoleaks and how to avoid them when these devices are used.

Using a single hybrid device such as the E-vita OPEN or the Thoraflex device makes it possible to treat aortic pathology extending into the descending thoracic aorta in a 1- or 2-stage intervention. The single-stage intervention has the competitive advantage of not requiring a second exposure to anesthetics, and no waiting period for the second stage, which could risk rupture or loss to follow-up. Disadvantages of this extensive procedure include the need to use both a hybrid room and fluoroscopy (to identify the level of the visceral vessels). Using fluoroscopy during circulatory arrest cases and using the special

From the Department of Cardiovascular Surgery, Texas Heart Institute, Houston, Tex; and the Division of Cardiothoracic Surgery, Michael E. DeBakey Department of Surgery, Baylor College of Medicine, Houston, Tex.

Disclosures: Dr Preventza consults for Terumo Aortic and for W. L. Gore \& Associates, Inc. Dr Cekmecelioglu reported no conflicts of interest.

The Journal policy requires editors and reviewers to disclose conflicts of interest and to decline handling or reviewing manuscripts for which they may have a conflict of interest. The editors and reviewers of this article have no conflicts of interest.

Received for publication June 10, 2020; revisions received June 10, 2020; accepted for publication June 12, 2020; available ahead of print June 20, 2020

Address for reprints: Ourania Preventza, MD, Division of Cardiothoracic Surgery,

Michael E. DeBakey Department of Surgery, Baylor College of Medicine, BCM

390, One Baylor Plaza, Houston, TX 77030 (E-mail: preventz@bcm.edu).

JTCVS Techniques 2020;3:23-4

2666-2507

Copyright (C) 2020 The Authors. Published by Elsevier Inc. on behalf of The American Association for Thoracic Surgery. This is an open access article under the CC BY-NCND license (http://creativecommons.org/licenses/by-nc-nd/4.0/).

https://doi.org/10.1016/j.xjtc.2020.06.012

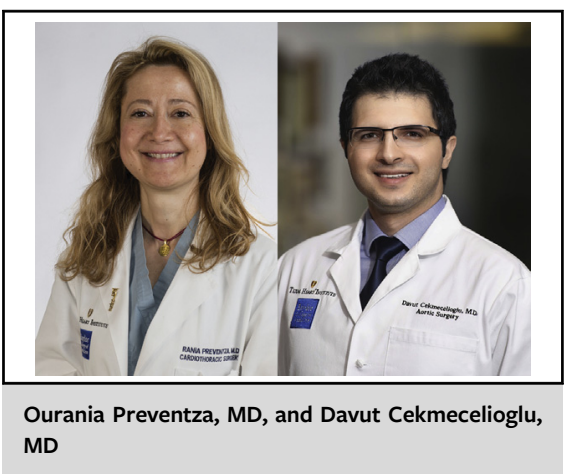

CENTRAL MESSAGE

Given that one-piece hybrid devices are relatively new, careful application is advisable, and the choice between a 1- or 2-stage approach should be individualized to the patient.

hybrid-suite operating table during these cases can be cumbersome.

In addition to these logistical issues, the most important disadvantage of the single-stage procedure is the risk of spinal cord ischemia ( $\mathrm{SCI}$ ) when significant coverage of the descending thoracic aorta (DTA) is required. In our recent meta-analysis of FET in more than 3000 patients, SCI was associated with coverage $>10 \mathrm{~cm}$. The 1 - and 2-stage approaches were not compared in that series. ${ }^{2}$ Interestingly, none of the patients in the current series ${ }^{1}$ experienced permanent or transient paraplegia or any degree of SCI.

Blood pressure (BP) management during the proximal repair differs from BP management during thoracic endovascular aortic repair (TEVAR) of a descending thoracic aneurysm with coverage of the entire DTA. During the proximal and arch repair, mean BP 60 to $80 \mathrm{~mm} \mathrm{Hg}$ may be sufficient to prevent bleeding from the fresh anastomoses. In contrast, during TEVAR, this mean BP is inadequate, and we drive the pressure to much greater levels when the entire DTA is covered. So in a single-stage approach, we avoid using one of the most important preventive measures for SCI. In addition, cerebrospinal fluid drainage, another measure to prevent SCI, is not widely used during proximal ascending and arch repair because drain insertion in a fully heparinized patient can cause hematoma.

The 2 -stage repair requires 2 procedures and a second use of anesthetics, as well as incurring the risk of rupture between stages. However, the proximal procedure is much 
less extensive. During the second stage, the patient's BP can be kept high enough to avoid SCI, and cerebrospinal fluid drainage can be also used. In addition, the variety of available sizes and devices reduces the risk of endoleak. For these reasons, we prefer the 2-stage approach.

Endoleak and aneurysm sac expansion after TEVAR can have a variety of causes: improper sizing, inadequate healthy sealing zone size, use of devices outside of the indications for use, and use of devices for applications in which its long-term durability has not been proven and that are not the standard of care. Tortuosity of the aortic arch or the entire thoracoabdominal aorta, the diameter of the distal and proximal landing zones, and overaggressive or underaggressive ballooning of the endograft can contribute to inadequate sealing, endoleak, and aneurysm sac expansion. The authors make recommendations for avoiding endoleaks and sac expansion. Their rate of reintervention is alarming, which makes "single-stage approach" a misnomer.

Although there are no clear recommendations for distal FET stent sizing, we extrapolate this knowledge from the current recommendations for aneurysmal disease and
TEVAR. Certainly, in a single-stage FET, sizing could be challenging with the premade one-piece devices available on the market. The authors' recommendations of $>10 \%$ oversizing and $>30$-mm distal seal, although arbitrary, are helpful and are not far from the classic TEVAR guidelines in patients with aneurysm. However, with the current Thoraflex device, the recommended distal seal is more than $40 \mathrm{~mm}$ of healthy aorta, with a recommended oversize of $15 \%$ to $25 \%$, slightly different from what the authors recommend for TEVAR but similarly arbitrary.

Given the relative newness of these hybrid devices, careful application is advisable. The choice between a 1- or 2stage approach should be individualized to the patient, and experience with endovascular techniques and appropriate sizing is required to avoid endoleaks.

\section{References}

1. Kandola S, Abdulsalam A, Field M, Fisher RK. Frozen elephant trunk repair of aortic aneurysms: how to reduce the incidence of endoleak and reintervention. J Thorac Cardiovasc Surg Tech. 2020;3:13-20.

2. Preventza O, Liao JL, Olive JK, Simpson K, Critsinelis AC, Price MD, et al. Neurologic complications after the frozen elephant trunk procedure: a metaanalysis of more than 3000 patients. J Thorac Cardiovasc Surg. 2020;160: 20-33.e4. 